\title{
Smart City for Future: Design of Data Acquisition Method using Threshold Concept Technique
}

\author{
Deepak NR, Thanuja N
}

\begin{abstract}
The massive deployment of Internet of Things (IoT) is allowing Smart City projects and initiatives all over the world. Whereas IOT deals with intricate systems that integrates multiple disperse components towards their synergetic use. Due to resource constraints, there will be a problem in the future to provide all the services to the residents. To continue to serve and improve the standard of living of the growing population, it is necessary to develop smart cities. The Smart City aims to make optimal and sustainable use of all resources, while maintaining an appropriate balance between social, environmental and economic costs. The wireless sensors are attached to street lamp, water tank, dustbin. Sensors are then attached to Arduino microcontroller board where each and every necessary parameter for city are monitored and updated to cloud. The cloud is connected with Blynk server in turn which is connected to build in Blynk application of user's Android phone. The technology we use is IOT in this model a system of interconnected smart modules is developed where each and every parameter necessary for a city is monitored and updated to the cloud. Emphasis is given on how sensing and communication technologies of IOT can effectively be used in smart city monitoring. The project aims at developing a system which facilitates aids in collection of data with the help of interconnected modules consisting of multiple sensors useful for smart city monitoring. Further this project also includes controlling of some parameters like water, light and dust bin.
\end{abstract}

Keywords: Street lamp, Water tank, Dustbin, Blynk App

\section{I.INTRODUCTION}

\section{Internet of Things (IoT) is a cutting-edge} conversational pattern achieved withinside the present day Which can be prepared with microcontrollers, transceivers for everyday life objects digital Conversation, and appropriate protocols stacks in a notable way with the cause of permitting them to talk with everyone Separate and with customers, which have become a vital part of the Internet. IoT The concept, therefore, objectives to make the Internet even easier. Also, get the proper through ease's permission Access and engage with a big array of devices that include, for example, the home.

Manuscript received on October 04, 2021.

Revised Manuscript received on October 25, 2021.

Manuscript published on October 30, 2021.

* Correspondence Author

Deepak NR*, Professor, Department of Computer Science \& Engineering, HKBK College of Engineering, Bangalore (Karnataka), India, E-mail. deepaknrgowda@gmail.com

Thanuja N, Asst. Prof., Department of Computer Science \& Engineering, Bangalore Institute of Technology, (VTU), Bangalore (Karnataka), India. E-mail. nthanuja@bit-bangalore.edu.in

(c) The Authors. Published by Blue Eyes Intelligence Engineering and Sciences Publication (BEIESP). This is an open access article under the CC BY-NC-ND license (http://creativecommons.org/licenses/by-nc-nd/4.0/)
Appliances, Surveillance Cameras, Surveillance Sensors, Actuators, Displays, Vehicles, and so on, IoT Will purpose the development of a number of applications that likely rate big sums of lease and Fashion the facts generated thru such objects to provide new services to citizens, companies, and Public Administration [1]. People who live in smart cities require smart practices to keep up with them. Serious competition. that include illumination, internet net web page online net internet site on- line vacationer control, consisting of a couple Of cities, energy consumption and pollutant control. The biggest purpose for smart metropolis Change the way we have a look at subjects. In relation to the different factors in which IoT is made up Rule we are in a characteristic to mention that each day progresses from the most reliable to the most Complex human emotions, IoT may have an impact on them all. Usually from Smart Town Citizens will often experience the applications and the underlying environment [2] City avatar A type of very complex systems: civil engineering infrastructure, ICT infrastructure, social so Network, economic community, etc. All systems require big manipulation effort (monitoring, reporting and intervention) to ensure seamless general not unusual place usual overall performance of all Applicable activities. Examples of such systems are public delivery systems, powered via energy. Power generation, water and fuel delivery systems, waste management, and protection the infrastructure of the metropolis, in conjunction with roads and public parks. On a social and economic plane, there web sites of college buildings, antiquities and tourism are systems for manipulating Interest, for commerce, for clinical and elderly care etc. Mass adoption of IoT technology in immoderate cap potential to conquer more than one metropolis operations, beginning from capricious Mechanically percentage facts, negotiate and get proper of access to services on every occasion and A need everywhere can be an inherent characteristic of smart cities. With the sum of aggregate Real with digital for the duration of the globe, it could be feasible to efficiently connect, integrate and employ Information generated thru an ever-evolving massive variety of metropolis actors, gadgets and events. Considering the above, it's far very easy for cities to have a superb environment. IoT research, because of the truth they represent a totally dense techno- socioeconomic tool that would act as a Beneficial shipping of hard practical and non-beneficial requirements from some software program application Program domain [3] IoT plays an immoderatethreat alerting role.

\section{Published By:}

Blue Eyes Intelligence Engineering

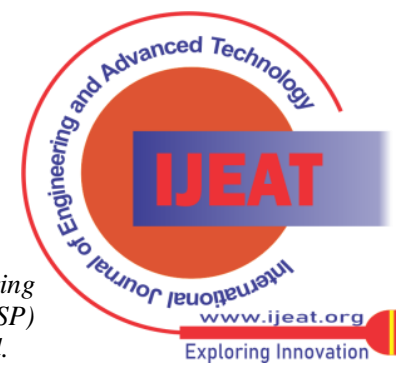




\section{Smart City for Future: Design of Data Acquisition Method using Threshold Concept Technique}

Smart home is Also organized with the protection of sending emails to customers and nearest. The next problem is Smart waste manipulate tool that permits municipal businesses to track the waste, and the release of bins, any toxic fuel line and video show device is better wasted. 1/3 characteristic Smart metropolis is smart street with naïve street lighting fixtures that can be Included with How online net internet site on- is a place solution that would aid govern web sites. wèb page site visitors go together with the go with the drift thru sensing Web net web page online net internet site on-line vacationer density. With the above features, speed Sensors are also system to show on street moderate furniture for a time body around Area upon motion detection withinside the presence of plenty much less natural medium, resulting in greater Naïve and energy saving environment [4].

The Internet of Things (IoT) is being used in a smart water tool. In step range one, the required duration is determined and achieved withinside the WDS thru reading and comparing Generation, device, rate and technology for assembling smart water system.

This famous show An IoT duration requirement with mixed technology for water distribution device. Also takes in takes the reputation of its blessings and disadvantages is based totally completely absolutely on literature review. Desire Skipping this step can diagnose the terrible desire for smart water system. Next step Using IoT for water distribution involves the want of the favored parameters.

At this step, the cutting-edge There are troubles at some level withinside the will of the parameters and some suitable suggestions: Compensated. Finally, the definition of crucial blessings for implementing IoT in smart water system is cited [5]. The tool is generally based totally mostly on Smart and Weather Adaptive Automatic Road Lights and Manipulate. Automation simplifies many troubles withinside the international economic tool withinside the same way as all Everyday life. It uses ultrapresent-day technology in LEDs due to the fact that the medium distribution Restore traditional street lamps in conjunction with HID lamps or immoderate tension sodium lamps etc.

A LED moderate furniture are a gift but because of it sever blessings Technologies collectively with advanced cuttingedge luminous efficiency, energy saving due to low protection rate, erratic coloration rendering index, prolonged start-up, and durability [6] In this letter they Sensor toolused to check waste level on dustbin and inform concerned people Authorization thru GSM/GPRS. The character, on the other hand, has a genuine right of $g$ because to the model's lack of automaticity proper of access to Locking bins and a completely unique bin, even though it's far very full. In addition, achieved near infrared Reflection (NIR) spectroscopy to pick out any shape of plastic waste and GSM production for the same chit chat. These are not truely RFID or GPS based totally completely surveillance systems at the moment. Smart method to implement on a citywide empire of times collectively with installing all-in- character GPS chip Trash bins [7] The factor of proper get proper of access to to dustbins. With the emergence of smart cities and greater modern generations, collectively with the Internet of Things (IoTs), which include RFID, environmental sensors, Actuator smartphones, wearable sensors, cloud computing and their applications on the town environments provide an opportunity to gather and manipulate metropolis facts on a big scale Information focus and desire building. Data from the device and/or a trendy reset of the gadgets also can moreover Include with Gift Town facts is stored thru Severa departments and community agencies and software program application applications have to be analyzed to assemble unique facts and understanding.

Like these Large-scale facts processing and storage can be accomplished in a cloud environment Meet the provider's requirements, for example, thru the response time to leaving Buyer questions Cloud based totally completely provision of truely infinite computational and storage facilities [8]. This paper proposes IoT based totally completely truely low-rate smart metropolis for the future Information Acquisition Approaches for the Use of Threshold Concept Techniques with an Advanced Android App for customers. This device can be used as a real time environment racking device.

The purpose Research to find out a private environment monitoring device embedded with an IoT device Calculate very low energy consumption. This tool is generally based totally mostly on IoT CAN Explores water ratio, running medium, waste manipulation in indoor environments collectively with home, art Work area for transmitting sensory facts to the patron of this Android software program application software, etc.

Which is named "Blynk App". Advanced which can be accessed thru internet connection. design Strategies and techniques are described in Phase II.

Experimental setup that includes simulation Format, hardware and software program application software program application implementation is in quick cited withinside the phase III. In step IV, the facts example is displayed. Finally, future art work and waste are investigated. During Phase V.

\section{DESIGN OF THE PROCEDURE}

Design of Process Implementation on a Keeping Aspect energy consumption, water manipulation, waste manipulation device is mainly immoderate rate as properly as the purpose is to get a long-term purpose for showing up on a big scale. It can be very crucial that net internet The net web page is optimized for big coverage at low fees online. This consequences in plenty much less rate and Effective solution for viewing the environment. The proper tool proposed proper right here is based totally completely It has fantastic vision and storage, absolutely on the transmission of environment variables Connected thru internet platform. The proposed device uses IoT to address the surrounding in real time software. LDR sensor, ultrasonic sensor and water go together with the go with the drift sensor are embedded in this tool to show Consistent facts to preserve the same vintage atmosphere.

Published By:

Blue Eyes Intelligence Engineering

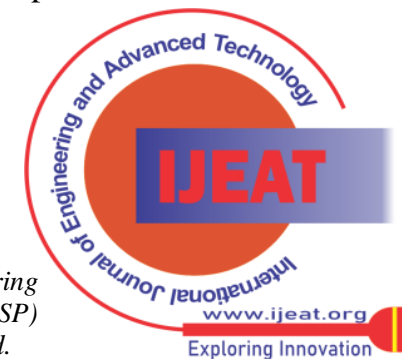




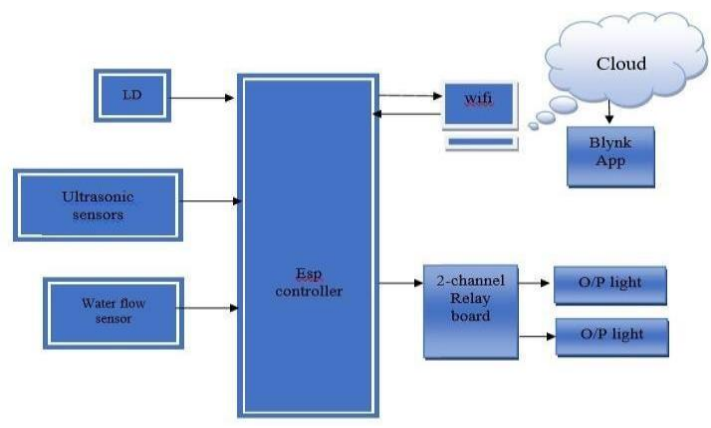

Fig 1. Block diagram of the proposed system

The proposed device is displayed It consists of LDR, ultrasonic and water thru an Android software program application software referred to as the Blynk app. go together with the go with the drift sensor. For the development of this tool, nodes may be stated due to the fact Hardware and software program application applications to gather and transmit facts will format the software program application to the server.

The node consists of a microcontroller ESP32. ESP controller has inter- connection WIFI and 2-channel relay board, in which it's far designed to alternate 2 immoderate energy Device from Arduino.

The 2-channel relay board need to have LEDs on the relay module indicating that Location of the Relay Whenever a relay is activated, the corresponding LED turns on.

In Suggestion Tool the Blynk app is mounted on the cloud to keep facts using the Internet and The Blynk app is hooked up for the duration of the Android software. Upgraded to an Android App Leaves the customers up the the front to test the accumulated facts. The format of the proposed device is confirmed withinside the block diagram confirmed in Figure 1

\section{PROPOSED SYSTEM ARCHITECTURE}

The proposed tool shapes the proposed model is cited withinside the most crucial Parts: Hardware implementation and software program application.

\section{A. Hardware Implementation}

The hardware implementation of the proposed device mainly includes: Microcontroller ESP32 It is notably identified for ultralow power consumption, power complete and characteristic-packed.

Microcontrollers commenced out to be used to have a take a take a look at and control facts from sensor. It sends facts to pre-jail Wi-Fi and a 2-channel relay board.

In us in the venture we have got were given 3 modules Light Module, Water Module, Dustbin Module.

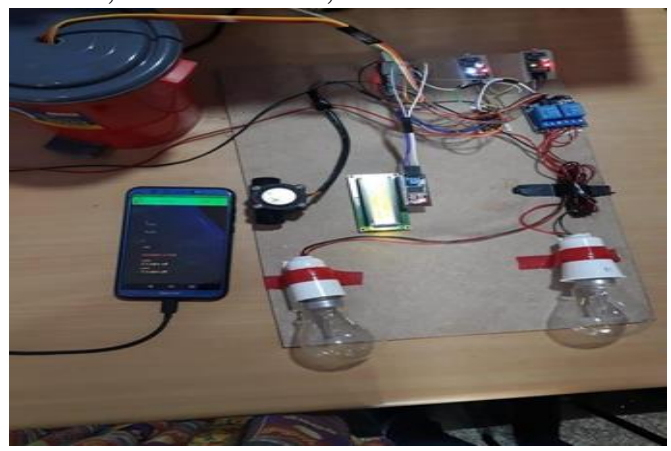

Fig 2. Hardware model 1.Light module

A Light Dependent Resistor (LDR) is a very unique shape of resistor whose resistance Price revisions depend upon the volume of the medium falling on it. It can be used to do diploma Ambient medium gift amount.

We use a digital LDR module. It has an on-board potentiometer that Used to set limits. If the moderator withinside the room is above a threshold, it gives a immoderate output signal.

Otherwise, it gives plenty much less output. Mechanically turns medium even as LDR sensor is detected are have become off and the LDR is not detected then routinely moderated.

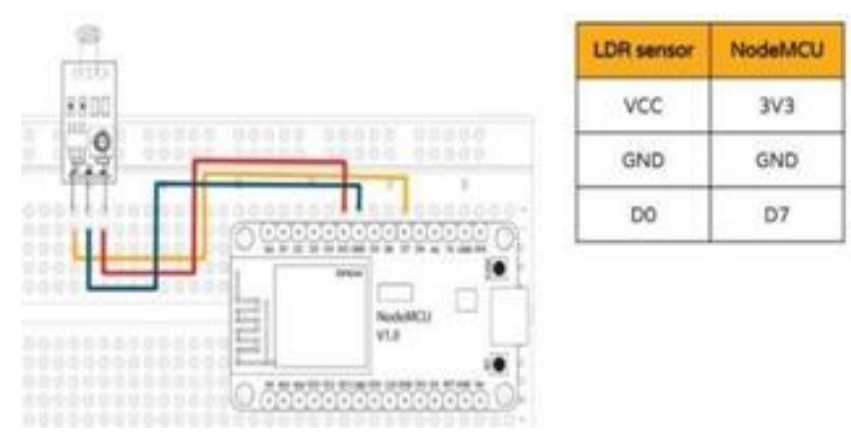

Fig 3. light model

\section{B. Water supply module}

Water Supply Module to reveal the amount of water being supplied and used, the rate of floatation of water desires to be measured. For this purpose, water go together with the go with the drift sensors are used. water go with the flow sensor Are installation on water distribution or pipe to diplize and calculate water go with the flow rate amount of water flowing. Connect the crimson cable output from the flowmeter to the $5 \mathrm{v}$ pin Connect the black cable output from the NodeMCU flowmeter to "Ground" on the NodeMQ. connect the yellow cable outputs (in my case the D2 pin) from the flowmeter to the control pin on the nodemQ.

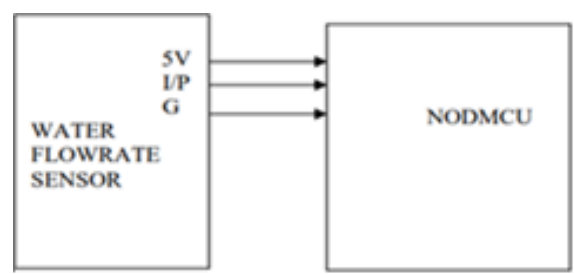

Fig 4. Water flow model

\section{Dustbin module}

Dustbin Module Ultrasonic Detected Waste Quantity Sensor and node MCU microcontroller. Internet is mounted using WIFI module and Notification may be given to that prisoner. Notification displayed amount in blynk app Trash and the reputation of that trash are displayed withinside the app. we are using node mcu Microcontroller as a controller. We are presenting energy supply to the controller through $9 \mathrm{~V}$ or $5 \mathrm{~V}$ DC battery or adapter. Since the controller and sensor require $5 \mathrm{~V}$. Ultrasonic sensor is used for stumbling the amount of waste and it continuously exams and sends facts to the controller.

Published By:

Blue Eyes Intelligence Engineering and Sciences Publication (BEIESP)

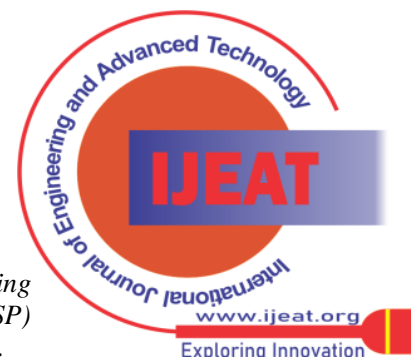


controller Analyzes the facts acquired thru the sensor and takes appropriate decisions. if there may be a dustbin The empty ultrasonic sensor detects and returns facts to the controller at the same time Updated facts withinside the Blynk app.

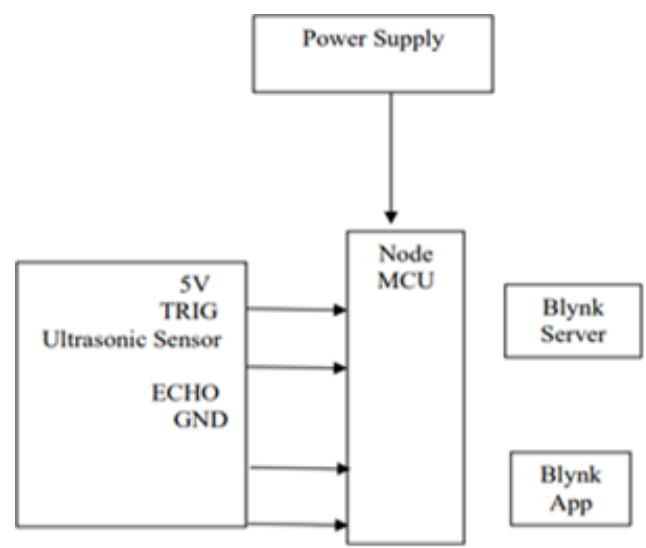

Fig 5. Dustbin model

\section{Software Implentation}

Software Implementation Mainly Blynk has a platform with IOS and Android apps to manipulate Arduino, Raspberry Pi and Liked on the internet. Arduino IDE (compiler) open deliver Arduino Software (IDE) makes it Clean to install writing code and upload to board. It runs on Windows, Mac OS, and. runs on Linux. Embedded $\mathrm{C}$ is a hard and fast of language extensions to the $\mathrm{C}$ programming language thru the $\mathrm{C}$ standards. Committee to address similarity troubles that exist amongst $C$ extensions for terrific Finally set up tool.

\section{Arduino IDE (compiler)}

Arduino Compiler or Arduino Software (IDE) Includes a text editor, a message area, a text console, a toolbar with buttons for writing code unusual location competencies and a number of menus. It connects to Arduino and Genuine hardware for convenience Make applications and talk to them. Arduino is an open-added electronics platform based totally completely mostly on easy-to-use hardware and software program application programmable. Arduino Forums Able to Study Input - Medium on a sensor, a finger on a button, or a Twitter message - and turn it into an output activating a motor, turning on a LED, publishing some troubles online.

\section{Embedded $C$}

Blynk is a toolset for all manufacturers, Badass inventors, designers, teachers, nerds and geeks who would love to use their smartphones Control Arduino, Raspberry-pi and similar electronics. we've got were given finished everything the hard art work of placing down an internet connection, building an app, and writing hardware code. Together Blynk, you without a doubt snap together a notable interface from the numerous widgets we provide, upload Example code on your hardware and revel in seeing the number one result in plenty much less than five minutes! its Works perfectly for novice makers and saves time for wicked geniuses. Blynk will art work with art All the famous structures and shields. We like to give you complete freedom, even to discover out the way to plug Blynk into your gift or new undertaking. You Blynk. can also revel in the benefits of cloud. Which, anyway, is loose and open- added. Blynk works over the Internet. So, most Effective and particular requires that your hardware can talk to the Internet.

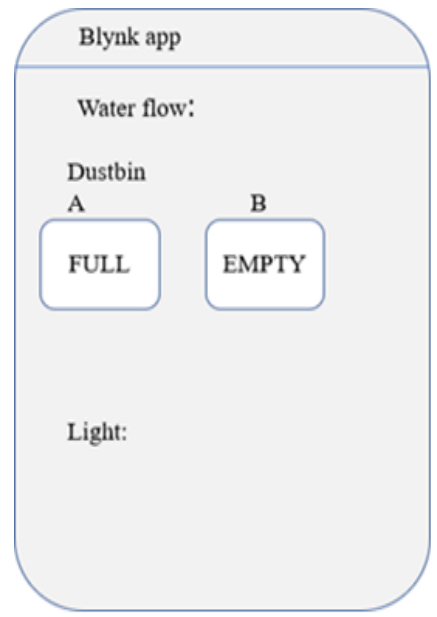

Fig 6. Blynk app

\section{Android Application}

App sheet is a platform to create apps for users using the data stored in cloud storage or in a spreadsheet. The app can be accessed from verity of devices such as smart phones and web browser.

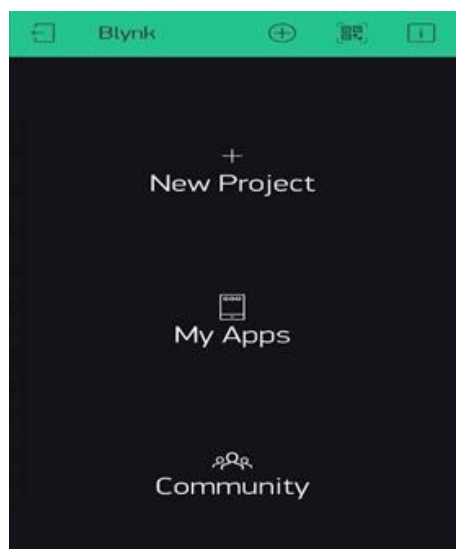

Fig 6. Mobile application

The monitoring application at first the data for different parameters such as various water flow, dustbin level light data collected using sensors later on the data was stored in spreadsheet. The spreadsheet was integrated with the app sheet platform which allowed customizing the front end of the application using various features offered by the app.

\section{IV.RESULT AND PERFORMANCE EVALUATION}

The overall performance of Development IoT Base Smart Town has been tested and showed via completing several experiments The Decide fig 7 shows the front-end up example of the sensor. the facts that became Found through device at terrific times. water go together with the go with the drift rate and dustbin level and whether or not or now no longer medium or moderate Whether or now no longer what is prepared to be displayed withinside the monitoring tool is now no longer on or off.

\section{Published By:}

Blue Eyes Intelligence Engineering

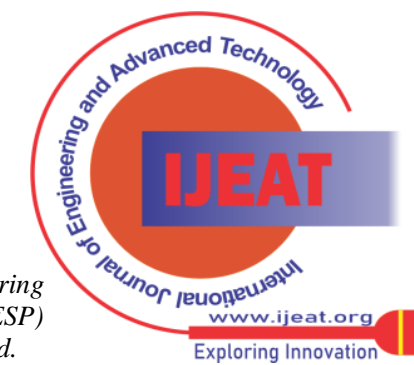


for someone unique pattern. Information on all three models is displayed through the server. these facts can be the patron receives the model's rate stored withinside the server for future assessment Server molecular app using IoT can display each model level this is confirmed in Figure 7.
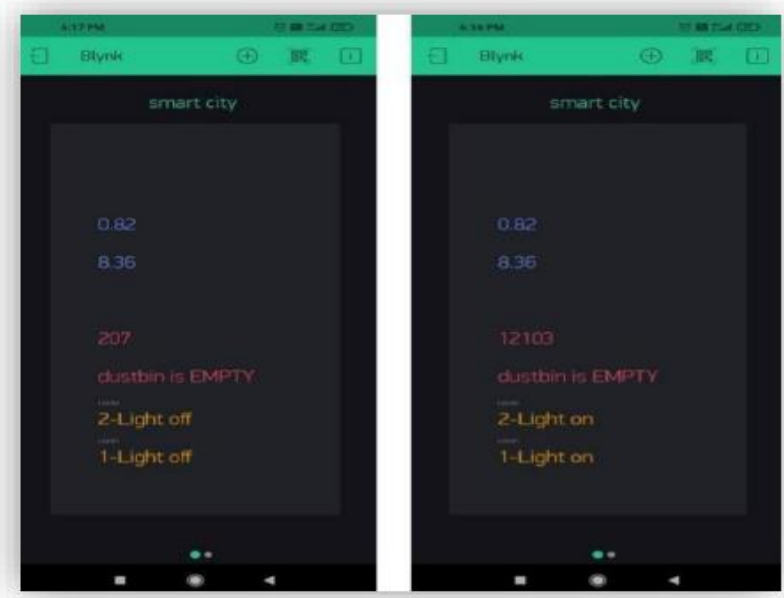

Fig 7. Result of light module
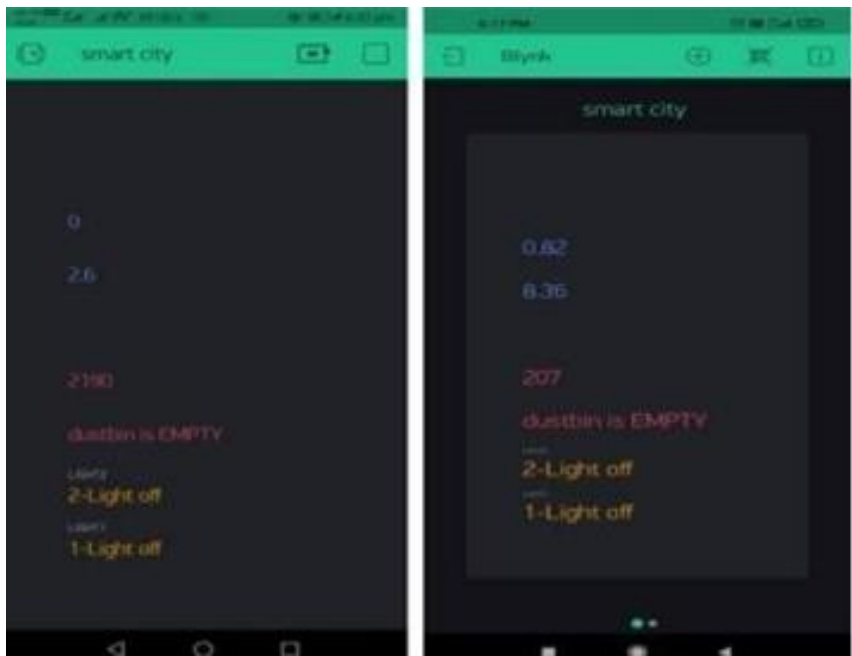

Fig 8. Result of waterflow module

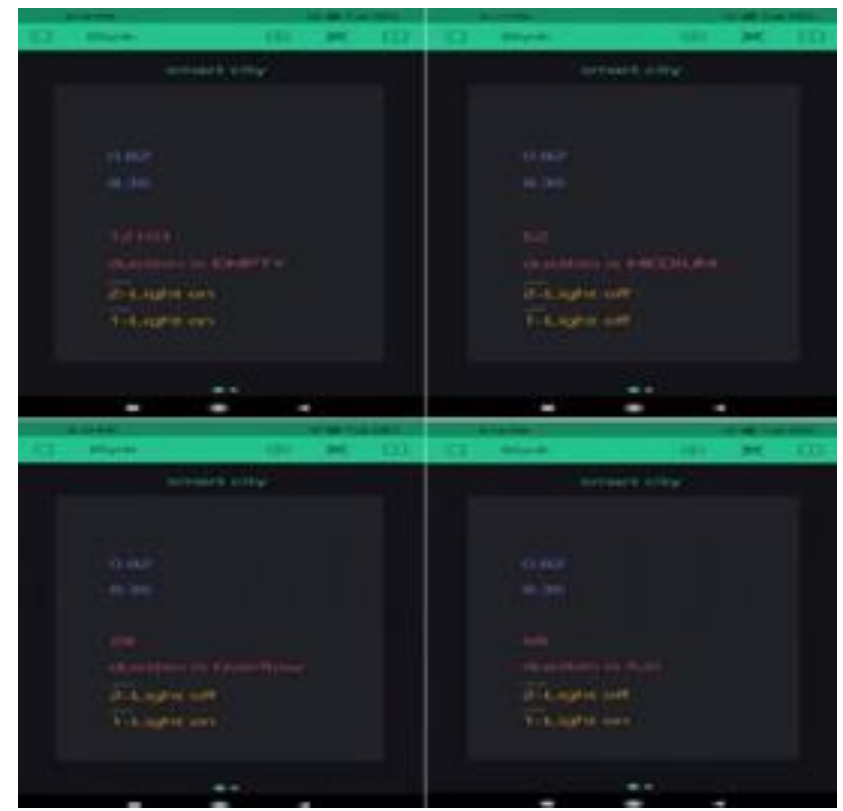

Fig 9. Result of dustbin module
All the sensors' data were displayed in the fig 7 for a total data level at each model and it will indicate the level of water and flow rate of water and dustbin level and light model it will indicates the on or off. Give withinside the front Sensor example instance facts All sensor facts displayed for the complete facts level on each model interior fig 8 and it's far going to propose water volume and water go together with the go with the drift rate and dustbin level and medium model is going to signify that on or off. Figure 8. Graphical example of sensor experimental facts the usual overall performance of the prototype model attests to its practicality, consistency and effectiveness. The rate assessment of the proposed device is displayed.

\section{트 Series nill 트 Series mid 틀 Series full}

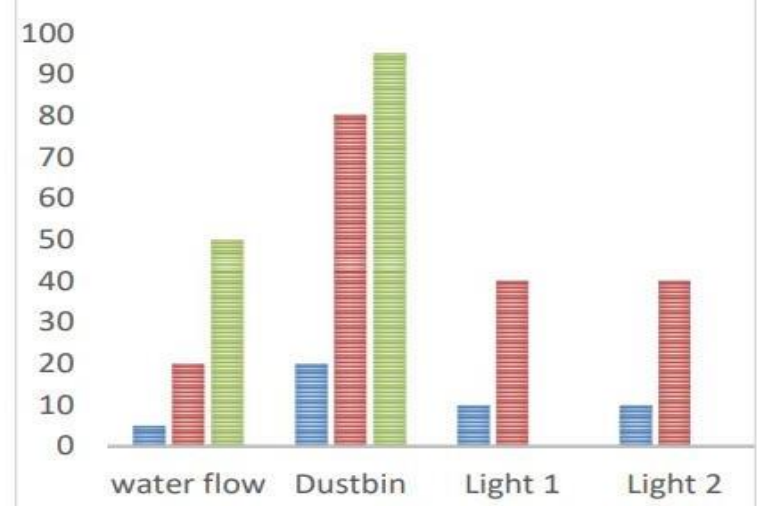

Fig 10. Graphical representation of sensors' data

\section{V.CONCLUSION}

In this paper IOT goes into this paper Getting rid of the boom of the fact's corporation. IoT remains in its early stages. plenty much less rate. hardware of the module achieved in IoT enabled systems has been designed and conceptualized for using this module Smart metropolis monitoring and control has been proposed. This undertaking objectives to boom a tool which permits to useful resource inside facet the collection of facts with the help of interconnected modulus which Include some parameters like water, medium and dustbin. The anxious character also can moreover display and Control anywhere inside facet the world. Can keep easy environment from time- to-time disposal of waste. go together with the go with the drift rate, to reveal the amount of water being supplied and used Water desires to be measured. For this a water go together with the go with the drift sensor is used. water go together with the go with the drift sensor Installation on the distribution or pipe of water to idolize the rate of water goes with the flow and calculate the amount Water flowed. Mechanically turns on medium and turns off even as iot the facts corporation is casting off a boom. IoT remains in its initial level. A's hardware the low-rate module achieved in IoT enabled systems has been designed and conceptualized for its use. A module for smart metropolis monitoring and control has been proposed.

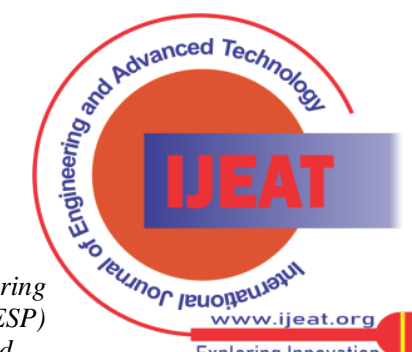

Blue Eyes Intelligence Engineering and Sciences Publication (BEIESP) (C) Copyright: All rights reserved. Exploring Innovation 


\section{FUTURE PERSPECTIVE}

Hopefully, the intention of Smart City is to make the excellent and sustainable use of all resources While maintaining a particular balance amongst social, environmental and economic fees. The most crucial pointers that define smart cities include goodness of the environment, power, Water and waste water, transportation and vicinity Site web page site visitors, facts and interaction systems, particular living, government, economics. human resources, housing and land use fatherland Security and emergency preparedness. The hardware of the low-rate module achieved in IoT enabled systems is has been designed and conceptualized to use this module for smart metropolis monitoring and control as proposed. One can keep a easy environment via eliminating the waste from time to time. to reveal the amount of water being supplied and used, the rate of floatation of the water desires to be measured. water go together with the go with the drift for this sensor is used. The water go together with the go with the drift sensor is set up on the water supply or pipe the rate of floatation of water and calculate the volume of water that has flowed. have become routinely moderate, which saves power

\section{REFERENCES}

1. J. Gubbi and R. Buyya, "Internet of things (IOT): A vision, architectural elements, and future directions," Future Generation Computer Systems 29, pp. 1645-1660, 2013.

2. T-Y Chen, H-W Wei, N-L Hsu, and W-K Shih, "A IOT application of safe building in IPV6 network environment," IEEE 37th Annual Computer Software and Applications Conference, pp. 748-753, 2013.

3. A. Monzon, "Smart cities concept and challenges bases for the assessment of smart city projects," IEEE Smart cities and Green ICT Systems (SMARTGREENS), pp. 1-11, May 2015.

4. A. Zelenkauskaite, N. Bessis, S. Sotiriadis, and E. Asimakopoulou, "Interconnectedness of complex systems of internet of things through social network analysis for disaster management," Intelligent Networking and Collaborative Systems (INCoS), IEEE, pp.503- 508, 2012

5. Radhakrishnan, V., \& Wu, W. (2018). IoT Technology for Smart Water System. 2018 IEEE 20th International Conference on High Performance Computing and Communications; IEEE 16th International Conference on Smart City; IEEE 4th International conference on Data S cience and Systems HPCC/SmartCity/DSS). oi:10.1109/hpcc/smartcity/dss.2018.00246 -smart water system

6. Zanella, A., Bui, N., Castellani, A., Vangelista, L., \& Zorzi, M. (2014). Internet of Things for Smart Cities. IEEE Internet of Things Journal, 1(1), 22-32. doi:10.1109/jiot.2014.2306328 - IOT for smart city

7. Rajab, H., \& Cinkelr Theodoridis, E., Mylonas, G., \& Chatzigiannakis, I. (2013). Developing an IoT Smart City framework. IISA 2013. doi:10.1109/iisa.2013.6623710-developing of iot

8. Rohit, G. S., Chandra, M. B., Saha, S., \& Das, D. (2018). Smart Dual Dustbin Model for Waste Management in Smart Cities. 2018 3rd International Conference for Convergence in Technology (I2CT). doi:10.1109/i2ct.2018.8529600-waste management

9. Dheena, P. P. F., Raj, G. S., Dutt, G., \& Jinny, S. V. (2017). IOT based smart street light management system. 2017 IEEE International Conference on Circuits and Systems (ICCS).

10. Khan, Z., Pervez, Z., \& Ghafoor, A. (2014). Towards Cloud Based Smart Cities Data Security and Privacy Management. 2014 IEEE/ACM 7th International Conference on Utility and Cloud Computing. doi:10.1109/ucc.2014.131 -cloud based smart city

11. Jain, S., Jatain, A., \& Bhaskar, S. (2019). Smart City Management System using IoT with Deep Learning. 2019 International Conference on Communication and Electronics Systems (ICCES). doi:10.1109/icces45898.2019.9002414- smart city is iot.

12. T. N and D. N R, "A Convenient Machine Learning Model for Cyber Security," 2021 5th International Conference on Computing Methodologies and Communication (ICCMC), 2021, pp. 284-290, Doi: 10.1109/ICCMC51019.2021.9418051.

\section{AUTHOR PROFILE}

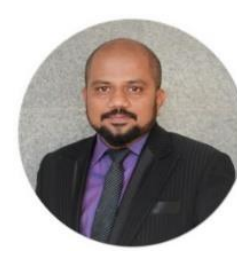

N. R. Deepak, M.Tech, Ph.D, FC-HRD, MISTE Motivating and Talented Sociological professor driven to inspire students to pursue academic and personal excellence. Consistently strive to create a challenging and engaging learning in which students become life-long scholar and learner. Deeply invested in achieving tenure through administrative service committee contributions and an accomplishment-oriented approach to teaching. With my Credentials and Enthusiasm to Placement \& Teaching Excellence for 15 years of experience, as a skilled, Research-Oriented, Placement Officer and Dedicated Teacher with a recent Doctorate Degree in Computer \& Information Sciences from VTU., I am well prepared to dedicate myself to the highest standards of instruction at your esteemed Institution and exceed your expectations for this position.

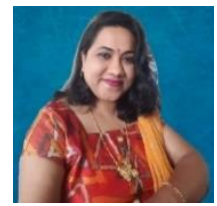

N. Thanuja, B.E., M.Tech, Assistant Professor and Researcher with $14+$ years of experience in teaching courses for both undergraduate and postgraduate levels at Bangalore Institute of Technology, Bengaluru. Experienced in Web Technology, Automata Theory, Machine Learning, Internet of Things and mentoring individuals. Dedicated, resourceful and goal-driven professional educator with a solid commitment to the academic growth. Committed to professional ethics and standards of practice. Utilizes creative teaching strategies to engage students fully in the learning process. Talent for bringing the classroom to life with real-world examples and innovative technology. Proven abilities in problem solving, people management and motivation. A self-starter with high energy enabling maximum and efficient work under pressure. Superior interpersonal and communication skills to foster meaningful relationships with students, staff and parents.
Published By:

Blue Eyes Intelligence Engineering and Sciences Publication (BEIESP) (C) Copyright: All rights reserved.

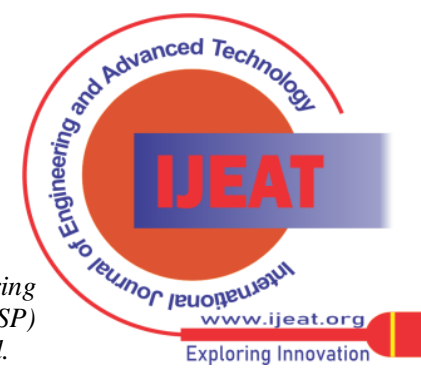

